Proceedings of the 5th Workshop on Quantum Chaos and Localisation Phenomena, Warsaw, Poland, May 20-22, 2011

\title{
Experimental Determination of the Autocorrelation Function of Level Velocities for Microwave Networks Simulating Quantum Graphs
}

\author{
M. Ławniczak, A. Borkowska, O. Hul, S. Bauch, and L. Sirko
}

Institute of Physics, Polish Academy of Sciences, al. Lotników 32/46, 02-668 Warszawa, Poland

The autocorrelation function $c(x)$ of level velocities is studied experimentally. The measurements were performed for microwave networks simulating quantum graphs. One and two ports measurements of the scattering matrix $\hat{S}$ necessary for determining $c(x)$ were realized for the networks possessing 5 and 6 vertices, respectively. The network with six vertices was fully connected. In the case of the networks with five vertices, additionally to the fully connected configuration, we measured the networks without the bond connecting input/output vertices. The obtained experimental results besides the autocorrelation function of level velocities, also the nearest-neighbor spacing distribution and parametric velocities distribution are compared to the predictions of random matrix theory and numerical results.

PACS: 05.45.Mt, 03.65.Nk

\section{Introduction}

The parameter-dependent autocorrelation function $c(x)$ of level velocities, where $x$ is the rescaled external parameter $X$, was introduced about 20 years ago by $[1,2]$. It was shown that the autocorrelation function $c(x)$ is a universal measure describing a quantum system which is classically chaotic.

The autocorrelation function $c(x)$ was studied theoretically in the case when the external parameter $X$ was a magnetic field [3-8], connected with a shape of a billiard [9] and in some other cases [10-13].

The real breakthrough in the experimental studies of this problem was possible when it was shown that microwave devices such as microwave cavities [14-27] and networks [28-35] may simulate quantum chaotic systems such as quantum billiards and graphs, respectively.

Their introduction extended substantially the number of systems which are used to verify wave effects predicted on the basis of quantum physics [36-39].

The experimental studies of the autocorrelation function $c(x)$ were presented e.g., in the papers [40, 41]. Recently, the connection of the parametric correlators of level velocities and the fidelity [42] resulted in revival of the interest in investigation of this effect. In $[43,44]$ numerical analysis of the parameter-dependent spectral statistics for quantum graphs, known as excellent paradigms of quantum chaos [12], is presented. In the mentioned papers, but also in many others, e.g., $[6,9,40,41]$, the departure of the autocorrelation function $c(x)$ of level velocities from the random matrix theory (RMT) prediction was reported. The discrepancies were accounted to some nonchaotic features of the investigated systems (e.g., existing of regular or quasiregular regions) or to localization phenomena in the case of quantum graphs.

\section{Experiment}

In this paper we present the results of the experimental study of the autocorrelation function of level velocities $c(x)$ for microwave networks simulating quantum graphs $[28-33,35]$ with preserved time reversal symmetry (TRS). We performed one and two ports measurements of the scattering matrix $\hat{S}$ for the microwave networks possessing 5 and 6 vertices, respectively. The network with six vertices was fully connected. In the case of the networks with five vertices, additionally to the fully connected configuration, we also measured the networks without the bond directly connecting input/output (cross-talking bond) vertices. The length of networks was chosen as an experimentally adjustable external parameter $X$ to generate level dynamics. In practice, the length of the chosen two bonds was changed simultaneously using two phase shifters.

Microwave network [28] with preserved TRS consists of $N$ vertices connected by bonds, e.g., coaxial cables. A coaxial cable is composed of an inner conductor of radius $r_{1}$ surrounded by a concentric conductor of inner radius $r_{2}$. The space between the conductors is filled with a homogeneous material having a dielectric constant $\varepsilon$. Thus the optical length of the cables is equal to the physical one times $\sqrt{\varepsilon}$. For a frequency $\nu$ below the onset of the next $\mathrm{TE}_{11}$ mode only the fundamental TEM mode can propagate inside a coaxial cable. The cut-off frequency of the $\mathrm{TE}_{11}$ mode is $\nu_{\mathrm{c}} \approx \frac{c}{\pi\left(r_{1}+r_{2}\right) \sqrt{\varepsilon}}=32.9 \mathrm{GHz}$ [45], where $r_{1}=0.05 \mathrm{~cm}, r_{2}=0.15 \mathrm{~cm}$ are the inner wire radii of the coaxial cable (SMA-RG402) conductors, and $\varepsilon \approx 2.08$ is the teflon dielectric constant [41, 46]. The phase shifters used to change the bonds' lengths are the reciprocal devices (undirected) exhibiting low insertion loss. In the experiment, ATM P1607 phase shifters with operating frequency range DC $18.6 \mathrm{GHz}$ and insertion loss less than $1 \mathrm{~dB}$ were applied. 
To measure the scattering matrix $\hat{S}$ of the networks we used typical experimental setup [32] with Agilent E8364B microwave vector network analyzer. The five (six) vertices networks were connected to the vector network analyzer through the leads - HP 85131-616 and HP 85131-617 flexible microwave cables - attached to the five (six) arms vertices in the case of two ports measurements. For one port measurements only the port no. 1 of the vector network analyzer was connected by the lead HP 85131-616 to the networks. It should be noticed that networks of the same "size" (number of vertices) used in the two ports and one port measurements are slightly different. The two port measurements require two vertices with the valency (number of bonds incident to a vertex) plus one, comparable to the other ones, whereas in the case of the one port measurements only one such a vertex is necessary. The scattering matrix was measured in the frequency range $0.1-9 \mathrm{GHz}$.

In order to investigate the autocorrelation function $c(x)$ of level velocities unfolding of the energy levels $E_{i}(X)=k_{i}^{2}(X)\left(\frac{\hbar^{2}}{2 m}=1\right)$, where $k_{i}(X)$ is a wave vector, and the external parameter $X$ is necessary to eliminate system-dependent features of the spectra. Instead of the original energy levels $E_{i}(X)$ one obtains the unfolded energies

$$
\xi_{i}(X)=N_{\mathrm{av}}\left(E_{i}(X)\right),
$$

where $N_{\text {av }}\left(E_{i}(X)\right)$ is the average number of energy levels with the mean level spacing $\Delta=1$ and for quantum graphs is given by the formula [47]

$$
N_{\mathrm{av}}\left(E_{i}(X)\right)=\frac{\sqrt{E_{i}(X)} L}{\pi}+\frac{1}{2} .
$$

The generalized conductance [2]

$$
C(0)=\left\langle\left(\frac{\partial \xi_{i}(X)}{\partial X}\right)^{2}\right\rangle,
$$

where $\langle\ldots\rangle$ is the averaging over the energy levels was used to unfold the parameter $X$ :

$$
x=\sqrt{C(0)} X \text {. }
$$

The autocorrelation function $c(x)$ of level velocities $[1,4]$ is defined as follows:

$$
c(x)=\left\langle\frac{\partial \xi_{i}}{\partial \bar{x}}(\bar{x}) \frac{\partial \xi_{i}}{\partial \bar{x}}(\bar{x}+x)\right\rangle .
$$

The averaging $\langle\ldots\rangle$ is over the parameter $x$ and energy levels. It is worth pointing out that the autocorrelation function $c(x)$ of level velocities correlates only velocities of the same energy level.

\section{Results}

The length of the six vertices network was changed from $4.140 \mathrm{~m}$ to $4.275 \mathrm{~m}$ in 45 equal steps $(1.5 \mathrm{~mm}$ per bond) using the phase shifters in the two of 15 bonds. 10414 parametric velocities were determined.

In Fig. 1 the experimental results for $c(x)$ are compared with the RMT and numerical predictions. The

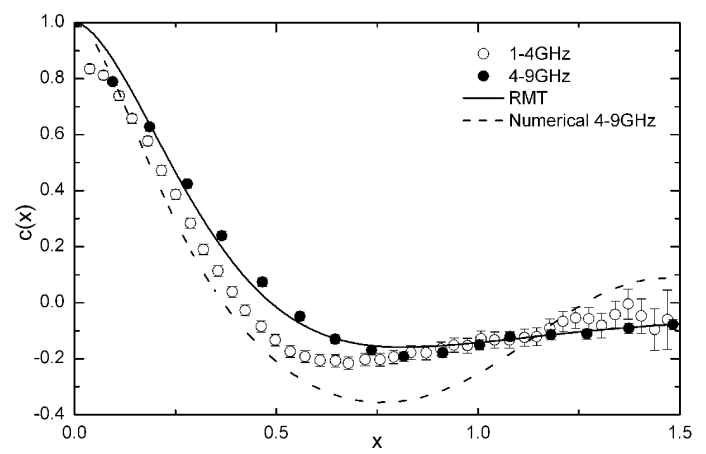

Fig. 1. The autocorrelation function $c(x)$ for the six vertices network. The experimental results (empty and full dots for results in the frequency range 1-4 and 4-9 GHz, respectively) are compared to the RMT (solid line) and numerical (dashed line) predictions.

empty and full circles represent the experimental results in the frequency window 1-4 and 4-9 GHz, respectively. The RMT and numerical predictions in the frequency window 4-9 GHz are denoted by solid and dashed lines, respectively.

The nearest-neighbor spacing distribution (NNSD) compared with RMT predictions for chaotic systems with preserved (Gaussian orthogonal ensemble - GOE) and broken (Gaussian unitary ensemble - GUE) TRS [48] is shown in Fig. 2.

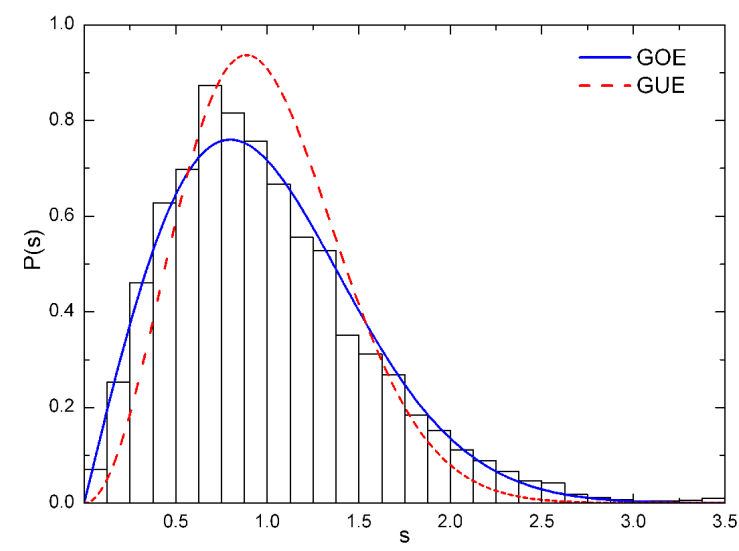

Fig. 2. The nearest-neighbor spacing distribution (NNSD) for the six vertices network compared with RMT predictions for chaotic systems with preserved (GOE) and broken (GUE) time reversal symmetry in the frequency range 1-9 GHz. The experimental data are represented by bars. The theoretical predictions for GOE and GUE are represented by solid (blue online) and dashed line (red online), respectively.

The parametric velocities distribution (PVD), which should be Gaussian for chaotic systems [49] is shown in Fig. 3. Parts (a) and (b) show the distributions for the frequency range $1-4$ and $4-9 \mathrm{GHz}$, respectively. In order to compare them to a Gaussian $P(\gamma)=$ 


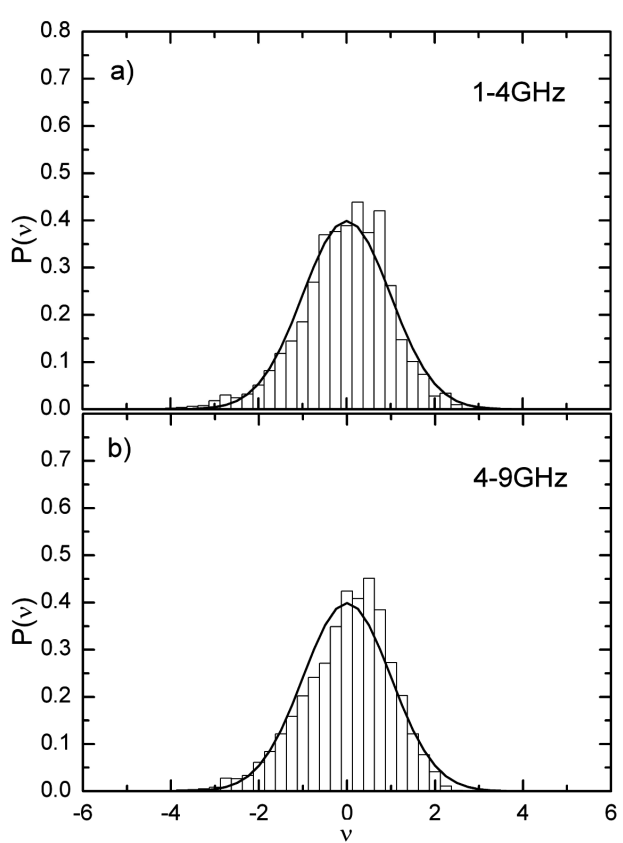

Fig. 3. The PVD for six vertices network. Parts (a) and (b) show the distributions for the frequency range 1-4 and 4-9 $\mathrm{GHz}$, respectively. The experimental data are represented by bars. The Gaussian $P(\gamma)=(1 / \sqrt{2 \pi}) \exp \left(-\gamma^{2} / 2\right)$ is denoted by solid line. $\sigma_{l}=0.035, \sigma_{h}=0.091$.

$(1 / \sqrt{2 \pi}) \exp \left(-\gamma^{2} / 2\right)$ parametric velocities were rescaled using the variance $\sigma^{2}=\left\langle\left(\frac{\partial \xi_{i}}{\partial \bar{x}}\right)^{2}\right\rangle$ :

$$
\nu_{i}=\frac{\partial \xi_{i} / \partial \bar{x}}{\sigma} \text {. }
$$

For the frequency range $4-9 \mathrm{GHz}$ the autocorrelation function $c(x)$ in Fig. 1, except small values of the unfolded parameter $x(0-0.15)$, follows quite well the RMT prediction. However, for the lower frequency window 1-4 GHz the discrepancy between the theory and experiment are visible till $x \approx 1$. This may be connected with the fact that the lower eigenvalues of the system are less chaotic than the higher ones. The experimental results of the nearest-neighbor spacing (Fig. 2) and parametric velocities (Fig. 3) distributions are in a reasonably good agreement with the theoretical predictions. It is worth to notice that the variance of the PVD for the higher frequency range $4-9 \mathrm{GHz}, \sigma_{h}=0.091$ (part (b) of Fig. 3) and is $\approx 2.6$ times bigger than the variance for the frequency range $1-4 \mathrm{GHz}\left(\sigma_{l}=0.035\right)$ (part (a) of Fig. 3). The origin of the observed discrepancy between the experimental and RMT predictions for the autocorrelation function $c(x)$ requires further investigation.

In the case of the five vertices networks, the one port and two ports measurements were performed for fully connected networks (ten bonds). Additionally, the two ports measurements for the network without the bond connecting the input/output five-arms vertices were completed. In the case of the one port configuration the two

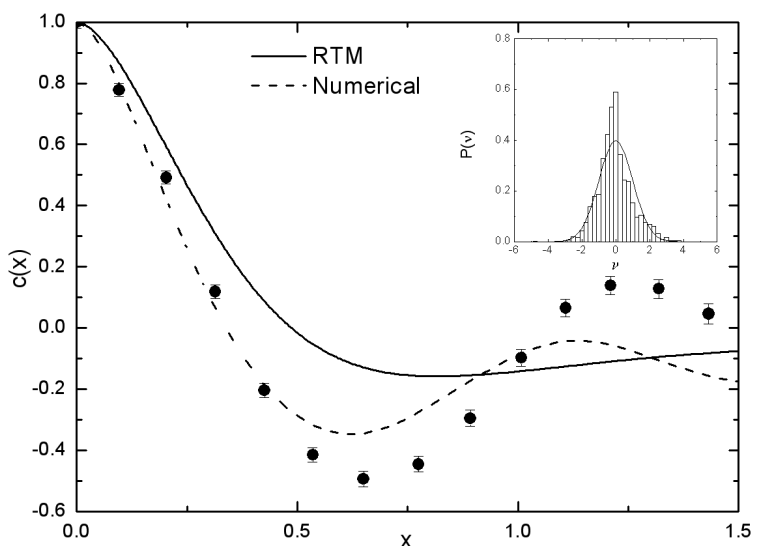

Fig. 4. The autocorrelation function $c(x)$ and PVD in the inset for one port measurement of five vertices fully connected network in the frequency range 4-9 GHz. The experimental, numerical results, and theoretical prediction for $c(x)$ are denoted by full circles, dashed, and solid line, respectively. In the inset bars represent the experimental results and the solid line denotes the Gaussian $P(\gamma)=(1 / \sqrt{2 \pi}) \exp \left(-\gamma^{2} / 2\right) . \sigma_{h}=0.110$.

measurements were performed. In the first one the overall length $3.022 \mathrm{~m}$ of the network was kept constant, while the lengths of the two of ten bonds were changed. The length of the one bond was increasing and the length of the other one was decreasing in 25 steps of $1.5 \mathrm{~mm} .4787$ parametric velocities $\frac{\partial \xi_{i}}{\partial \bar{x}}$ were obtained.

In Fig. 4 the autocorrelation function $c(x)$ is presented. Additionally, the parametric velocities distribution for the frequency range $4-9 \mathrm{GHz}$ is shown in the inset. The evident departure from the RMT prediction is seen for both, the experimental and numerical results. For $x>0.45$ the numerical results are closer to the theoretical curve than the experimental ones. Again, the experimental results for the higher frequency range are in better agreement with the theoretical prediction than the results for lower frequencies which are not shown here. It may be argued that these more evident departures of the experimental and numerical results from the RMT prediction than in the case of the six vertices network are caused by the fact that the five vertices network is less chaotic compared to the previous one [43]. The lengths variation of only two out of ten bonds may be the other reason of the observed discrepancies. This seems to be confirmed by the preliminary results (not shown here) of the measurements of the fully connected five vertices network, in which the lengths of three bonds were changed.

The NNSD distribution (see Fig. 5) is in a good agreement with the RMT prediction for GOE. The parametric velocities distribution (inset of Fig. 4) exhibits visible excess over the Gaussian nearby zero. The excess is much less profound than in the lower frequency range and again the variance of the PVD for higher frequency is $\approx 2.9$ times bigger than the variance for the lower one.

The two ports measurements were performed for the 


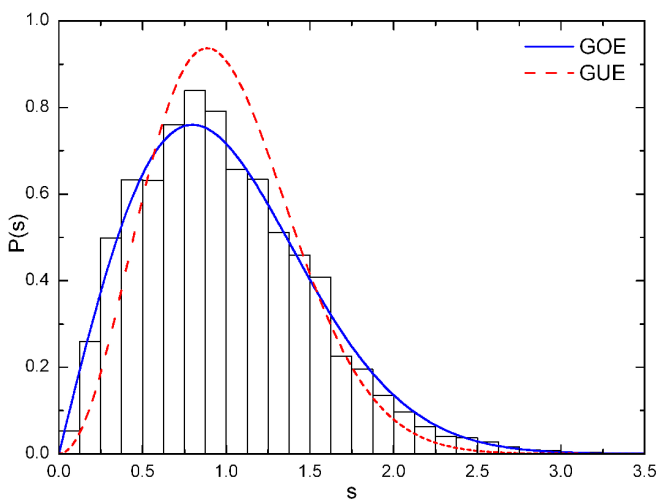

Fig. 5. The NNSD for one port measurement of five vertices fully connected network in the frequency range 0-9 GHz compared with RMT predictions for chaotic systems with preserved (GOE) and broken (GUE) time reversal symmetry. The experimental data are represented by bars. The theoretical predictions for GOE and GUE are represented by the solid (blue online) and dashed line (red online), respectively.

fully connected, 10-bond network and the network without the bond connecting input/output vertices which formed the 9-bond network. In the measurements the total length of the networks, $3.022 \mathrm{~m}$ and $2.465 \mathrm{~m}$, respectively, were kept constant. In order to generate the level dynamics the lengths of the chosen two bonds were changed by means of the phase shifters. While the length of one bond was increased the length of the other one was simultaneously decreased. Specifically, for the 10-bond network the lengths of the two bonds were changed in $45,1.5 \mathrm{~mm}$ long, steps while for the 9-bond network the bonds were changed in $23,1.5 \mathrm{~mm}$ long, steps. It allowed us to determine 6978 parametric velocities for the fully connected, 10-bond, network and 4553 for the other one.

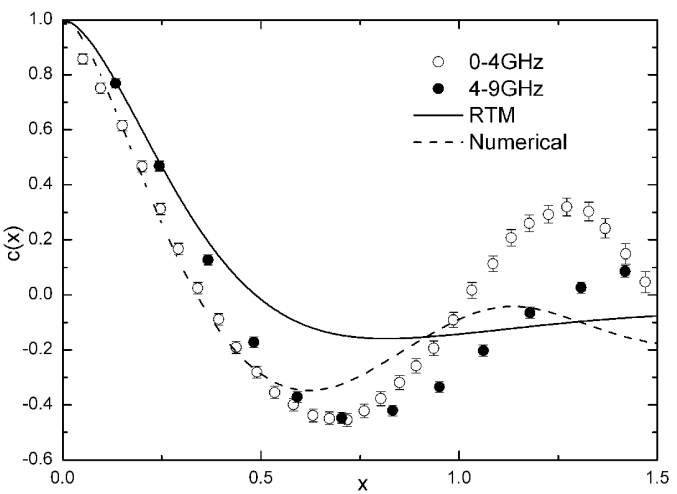

Fig. 6. The autocorrelation function $c(x)$ for two ports measurement of five vertices fully connected network. The experimental results (empty and full dots for results in the frequency range $1-4$ and $4-9 \mathrm{GHz}$, respectively) are compared to the RMT (solid line) and numerical (dashed line) predictions.

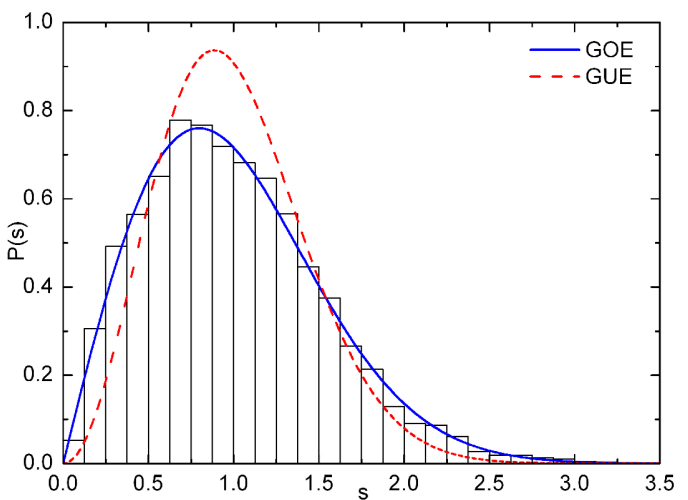

Fig. 7. The NNSD for two ports measurement of five vertices fully connected network in the frequency range 0-9 GHz compared with RMT predictions for chaotic systems with preserved (GOE) and broken (GUE) time reversal symmetry. The experimental data are represented by bars. The theoretical predictions for GOE and GUE are represented by the solid (blue online) and dashed line (red online), respectively.

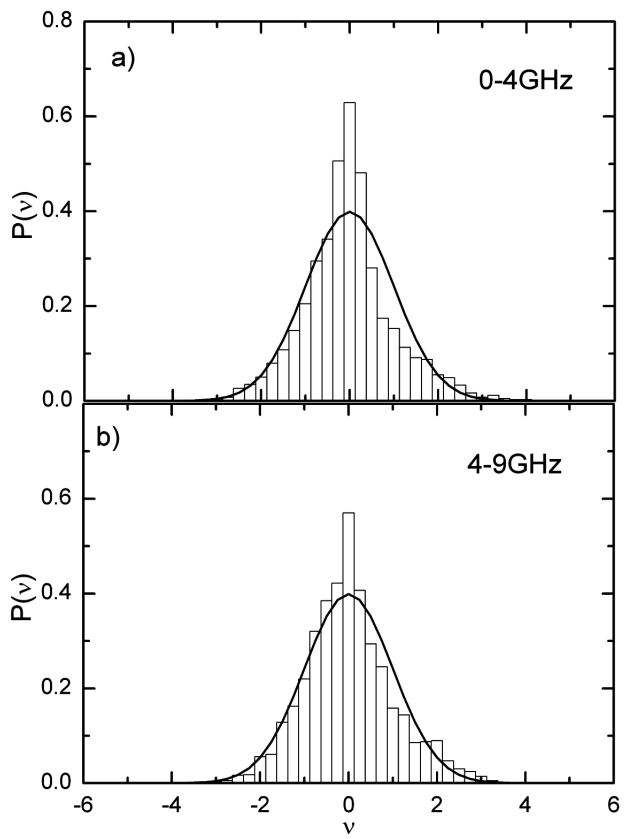

Fig. 8. The PVD for two ports measurement of five vertices fully connected network. Parts (a) and (b) show the distributions for the frequency range 0-4 and 4-9 GHz, respectively. The experimental data are represented by bars. The Gaussian $P(\gamma)=$ $(1 / \sqrt{2 \pi}) \exp \left(-\gamma^{2} / 2\right)$ is denoted by the solid line. $\sigma_{l}=$ $0.047, \sigma_{h}=0.118$.

The autocorrelation function $c(x)$ of the fully connected network is shown in Fig. 6. The presented results are clearly closer to the RMT prediction than the ones obtained in the one port measurements of the five vertices fully connected network. The possible explanation of this phenomenon is the following: because of the bigger absorption the width of the resonance curves are getting 
also bigger. Therefore, the positions of the resonances are determined with the bigger uncertainties which slightly influences the autocorrelation function $c(x)$.

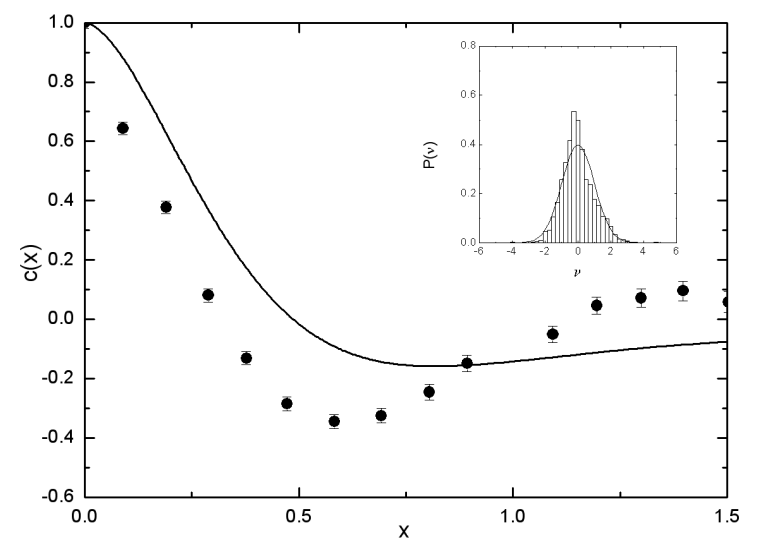

Fig. 9. The autocorrelation function $c(x)$ and PVD in the inset for two ports measurement of five vertices network without the bond connecting input/output five-arms vertices in the frequency range $4-9 \mathrm{GHz}$. The experimental results and theoretical prediction for $c(x)$ are denoted by full circles and solid line, respectively. In the inset bars represent the experimental results and the solid line denotes the Gaussian $P(\gamma)=$ $(1 / \sqrt{2 \pi}) \exp \left(-\gamma^{2} / 2\right) . \sigma_{h}=0.108$.

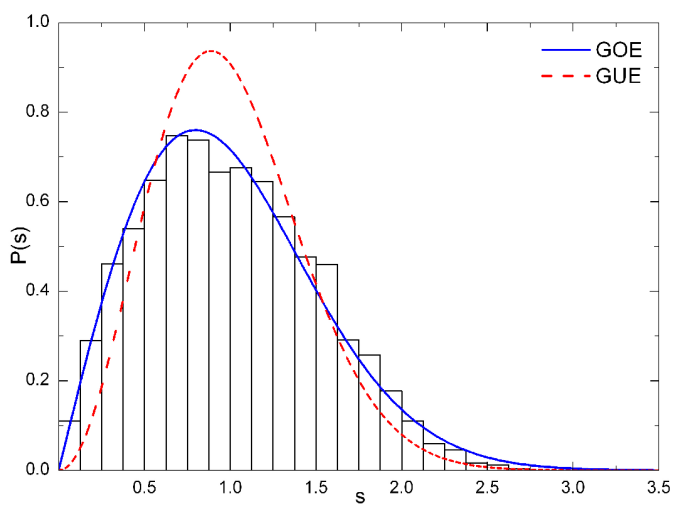

Fig. 10. The NNSD for two ports measurement of five vertices network without the bond connecting input/output five-arms vertices in the frequency range 0-9 GHz compared with RMT predictions for chaotic systems with preserved (GOE) and broken (GUE) time reversal symmetry. The experimental data are represented by bars. The theoretical predictions for GOE and GUE are represented by the solid (blue online) and dashed line (red online), respectively.

The NNSD and PVD for this network are shown in Figs. 7 and 8, respectively. They are also closer to RMT predictions than the ones obtained for the one port measurements. Specifically, the ratio of the $\sigma_{h} / \sigma_{l} \approx 2.5$ and is very close to the ratio for the six vertices network.

The results for the 9-bond network are rather surprising. One may expect that removing of the bond between the output/input vertices should profoundly eliminate the direct processes [50] and the results should not be worse than for the fully connected network.

However, the autocorrelation function $c(x)$ for the frequency range $4-9 \mathrm{GHz}$ presented in Fig. 9 shows bigger departure from the RMT prediction than the other ones.

The nearest-neighbor spacing distribution (Fig. 10) and the parametric velocities distribution (inset of Fig. 9) are definitely in better agreement with the RMT predictions than the autocorrelation function $c(x)$. The ratio of the $\sigma_{h} / \sigma_{l} \approx 3.0$ is the biggest in this case.

\section{Conclusions}

The experimental and numerical results presented in this paper for the microwave networks with $N=5$ and $N=6$ vertices show that the autocorrelation function $c(x)$, in contrast to the nearest-neighbor spacing distribution, departs from the RMT prediction. Bigger disagreement with the RMT prediction is observed for the graphs with smaller number of vertices. This observation is in the agreement with the theoretical paper [43] which additionaly predicts that for graphs with bigger number of vertices $(N \gg 6)$ autocorrelation function $c(x)$ departs from the RMT prediction due to localization phenomena. Further experimental investigations are necessary to clarify some discrepancies with the numerical results.

\section{Acknowledgments}

This work was partially supported by the Ministry of Science and Higher Education grant No. N N202 130239.

\section{References}

[1] X. Yang, J. Burgdörfer, Phys. Rev. A 46, 2295 (1992).

[2] B.D. Simons, B.L. Altshuler, Phys. Rev. B 48, 5422 (1993).

[3] A. Szafer, B.L. Altshuler, Phys. Rev. Lett. 70, 587 (1993).

[4] B.D. Simons, B.L. Altshuler, Phys. Rev. Lett. 70, 4063 (1993)

[5] M. Faas, B.D. Simons, X. Zotos, B.L. Altshuler, Phys. Rev. B 48, 5439 (1993).

[6] B.D. Simons, A. Hashimoto, M. Courtney, D. Klepner, B.L. Altshuler, Phys. Rev. Lett. 71, 2899 (1993).

[7] M.V. Berry, J.P. Keating, J. Phys. A 27, 6167 (1994).

[8] H. Bruus, C.H. Lewenkopf, E.R. Mucciolo, Phys. Rev. B 53, 6167 (1996).

[9] H. Bruus, C.H. Lewenkopf, E.R. Mucciolo, Phys. Scr. B T 69, 13 (1997).

[10] B.D. Simons, P.A. Lee, B.L. Altshuler, Phys. Rev. Lett. 72, 64 (1994).

[11] B. Dietz, M. Lombardi, T.H. Seligman, Phys. Lett. 215, 181 (1996).

[12] T. Kottos, U. Smilansky, Ann. Phys. 274, 76 (1999).

[13] I.E. Smolyarenko, B.D. Simons, Phys. Rev. E 67, 025202 (2003). 
[14] H.J. Stöckmann, J. Stein, Phys. Rev. Lett. 64, 2215 (1990).

[15] S. Sridhar, Phys. Rev. Lett. 67, 785 (1991).

[16] H. Alt, H.-D. Gräf, H.L. Harner, R. Hofferbert, H. Lengeler, A. Richter, P. Schardt, A. Weidenmüller, Phys. Rev. Lett. 74, 62 (1995).

[17] P. So, S.M. Anlage, E. Ott, R.N. Oerter, Phys. Rev. Lett. 74, 2662 (1995).

[18] U. Stöffregen, J. Stein, H.-J. Stöckmann, M. Kuś, F. Haake, Phys. Rev. Lett. 74, 2666 (1995).

[19] F. Haake, M. Kuś, P. Šba, H.-J. Stöckmann, U. Stöffregen, J. Phys. A 29, 5745 (1996).

[20] L. Sirko, P.M. Koch, R. Blümel, Phys. Rev. Lett. 78 2940 (1997)

[21] S. Bauch, A. Błędowski, L. Sirko, P.M. Koch, R. Blümel, Phys. Rev. E 57, 304 (1998).

[22] L. Sirko, Sz. Bauch, Y. Hlushchuk, P.M. Koch, R. Blümel, M. Barth, U. Kuhl, H.-J. Stöckmann, Phys. Lett. A 266, 331 (2000).

[23] R. Blümel, P.M. Koch, L. Sirko, Found. Phys. 31 269 (2001).

[24] Y. Hlushchuk, L. Sirko, U. Kuhl, M. Barth, H.J. Stöckmann, Phys. Rev. E 63, 046208 (2001).

[25] N. Savytskyy, S. Bauch, A. Błędowski, O. Hul, L. Sirko, Acta Phys. Pol. B 33, 2123 (2002).

[26] N. Savytskyy, O. Hul, L. Sirko, Phys. Rev. E 70, 056209 (2004).

[27] O. Hul, N. Savytskyy, O. Tymoshchuk, S. Bauch, L. Sirko, Phys. Rev. E 72, 066212 (2005).

[28] O. Hul, S. Bauch, P. Pakoński, N. Savytskyy, K. Życzkowski, L. Sirko, Phys. Rev. E 69, 056205 (2004).

[29] O. Hul, O. Tymoshchuk, Sz. Bauch, P.M. Koch, L. Sirko, J. Phys. A, Math. Gen. 38, 10489 (2005).

[30] O. Hul, S. Bauch, M. Ławniczak, L. Sirko, Acta Phys. Pol. A 112, 655 (2007).

[31] O. Hul, M. Ławniczak, S. Bauch, L. Sirko, Symp. Pure Math. 77, 595 (2008).

[32] M. Ławniczak, O. Hul, S. Bauch, P. Šeba, L. Sirko, Phys. Rev. E 77, 056210 (2008).
[33] M. Ławniczak, S. Bauch, O. Hul, L. Sirko, Phys. Scr. T 135, 014050 (2009).

[34] M. Eawniczak, O. Hul, Sz. Bauch, L. Sirko, Acta Phys. Pol. A 116, 749 (2009).

[35] M. Ławniczak, S. Bauch, O. Hul, L. Sirko, Phys. Rev. E 81, 046204 (2010)

[36] R. Blümel, A. Buchleitner, R. Graham, L. Sirko, U. Smilansky, H. Walther, Phys. Rev. A 44, 4521 (1991).

[37] M. Bellermannn, T. Bergemann, A. Haffmann, P. M. Koch, L. Sirko, Phys. Rev. A 46, 5836 (1992).

[38] L. Sirko, S. Yoakum, A. Haffmans, P. M. Koch, Phys. Rev. A 47, R782 (1993).

[39] L. Sirko, A. Haffmans, M.R.W. Bellermann, P.M. Koch, Europhysics Letters 33, 181 (1996).

[40] Y. Hlushchuk, A. Kohler, Sz. Bauch, L. Sirko, R. Blümel, M. Barth, H.-J. Stöckmann, Phys. Rev. E 61, 366 (2000).

[41] N. Savytskyy, A. Kohler, S. Bauch, R. Blümel, L. Sirko, Phys. Rev. E 64, 036211 (2001)

[42] H. Kohler, I.E. Smolyarenko, C. Pineda, T. Guhr, F. Leyvraz, T.H. Seligman, Phys. Rev. Lett. 100, 190404 (2008).

[43] O. Hul, P. Šeba, L. Sirko, Phys. Rev. E 79, 066204 (2009).

[44] O. Hul, L. Sirko, Phys. Rev. E 83, 066204 (2011).

[45] D.S. Jones, Theory of Electromagnetism, Pergamon Press, Oxford 1964, p. 254.

[46] K.H. Breeden, A.P. Sheppard, J. Microwave, 10, 59 (1967), K.H. Breeden, A.P. Sheppard, Radio. Sci. 3, 205 (1968).

[47] T. Kottos, U. Smilansky, Phys. Rev. Lett. 79, 4794 (1997).

[48] M.C. Gutzwiller, Chaos in Classical and Quantum Mechanics, Springer-Verlag, New York 1990.

[49] P. Lebœuf, M. Sieber, Phys. Rev. E 60, 3969 (1999).

[50] S. Hemmady, X. Zheng, T.M. Antonsen Jr., E. Ott, S.M. Anlage, Acta Phys. Pol. A 109, 65 (2006). 